Preprint typeset in JHEP style - HYPER VERSION

May, 2004

HIP-2002-47/TH

NORDITA-2002-67 HE

hep-ph/0210124

\title{
Subprocess Size in Hard Exclusive Scattering
}

\author{
Paul Hoyer ${ }^{1}$, Jonathan T. Lenaghan ${ }^{2,3}$, Kimmo Tuominen ${ }^{4,5}$ and Carsten Vogt ${ }^{4}$ \\ ${ }^{1}$ Department of Physical Sciences and Helsinki Institute of Physics \\ POB 64, FIN-00014 Helsinki University, Finland \\ ${ }^{2}$ Department of Physics, University of Virginia \\ 382 McCormick Rd., Charlottesville, VA 22903, USA \\ ${ }^{3}$ Niels Bohr Institute and ${ }^{4}$ Nordita \\ Blegdamsvej 17, DK-2100 Copenhagen, Denmark
}

\begin{abstract}
The interaction region of hard exclusive hadron scattering can have a large transverse size due to endpoint contributions, where one parton carries most of the hadron momentum. The endpoint region is enhanced and can dominate in processes involving multiple scattering and quark helicity flip. The endpoint Fock states have perturbatively short lifetimes and scatter softly in the target. We give plausible arguments that endpoint contributions can explain the apparent absence of color transparency in fixed angle exclusive scattering and the dimensional scaling of transverse $\rho$ photoproduction at high momentum transfer, which requires quark helicity flip. We also present a quantitative estimate of Sudakov effects.
\end{abstract}

KEYwords: Perturbative QCD, Exclusive reactions. 


\section{The dynamics of endpoint contributions}

In the Brodsky-Lepage (BL) framework of exclusive scattering [1, 2], the amplitude for a process $A+B \rightarrow C+D$ factorizes at large $t=\left(p_{A}-p_{C}\right)^{2}$ into a hard subprocess $a+b \rightarrow c+d$ times distribution amplitudes $\phi_{a / A}, \ldots$ for each external hadron. Here $a$ represents the valence Fock state of hadron $A$ (e.g., $a=u u d$ for the proton). The distribution amplitude is the valence Fock state amplitude at equal Light-Cone (LC) time, integrated over the relative transverse momenta of the partons up to a hard scale of $\mathcal{O}(\sqrt{-t})$. Hence all hadrons involved in the scattering are in transversally compact configurations. In the subprocess amplitude the momenta of the partons in each hadron are effectively parallel, their relative transverse momenta being negligible compared to the hard scale.

There are factorization theorems of various degree of rigour for QCD reactions. However, it should be kept in mind that for semi-exclusive processes and for hadronic projectiles in general, factorization is a strong assumption: no rigorous QCD proof exists. In this paper we qualitatively study which aspects of the data conform to general expectations when factorization is assumed. Our investigation builds on a physical picture rather than a precise formalism, and serves to indicate potential problems and possible solutions. This will hopefully guide the development of a more systematic formalism in future work.

The assumed factorization framework may indeed fail due to endpoint contributions in the integration over the longitudinal momentum fractions of the quarks [2, 3]. The longitudinal momentum of a quark with fraction $z \lesssim \Lambda_{\mathrm{QCD}} / p^{+}$of its parent hadron momentum $p^{+}$(in a frame where the hadron moves fast along the $z$-axis) is no larger than its transverse momentum. Hence the quark is isotropically distributed in momentum space and outside the light-cone formed by the fast quarks of the subprocess. The virtualities of subprocess propagators decrease with $z$, allowing hadron Fock states of large transverse size to contribute near the endpoints.

The lifetime $\tau$ of a Fock state in a parent hadron of high momentum $p^{+}=E+p^{z}$ is inversely proportional to the difference between its energy and that of the hadron,

$$
\frac{1}{\tau} \simeq \sum_{i} E_{i}-E \simeq \sum_{i} \frac{k_{\perp i}^{2}+m_{i}^{2}}{z_{i} p^{+}}-\frac{M^{2}}{p^{+}},
$$

where $M$ is the mass of the parent. The second approximation between the ordinary and light-cone energy differences is valid when $z_{i} p^{+}=k_{i}^{+} \gg k_{\perp i}, m_{i}$ for all constituents $i$ and is thus not valid for Fock states in the endpoint region. The lifetime of the endpoint Fock states is $\sim 1 / \Lambda_{\mathrm{QCD}}$, which is short compared to the typical lifetimes of $\mathcal{O}\left(p^{+} / \Lambda_{\mathrm{QCD}}^{2}\right)$ of Fock states where all constituents have comparable momentum fractions $z_{i}$.

The nature of endpoint dynamics is illustrated by Deep Inelastic Scattering $(e p \rightarrow e X$, DIS). In the aligned jet (parton model) regime the virtual photon 
with $q^{+} \simeq 2 \nu$ splits asymmetrically into a $q \bar{q}$ pair, such that $z_{\bar{q}} \sim \Lambda_{\mathrm{QCD}}^{2} / Q^{2}$ and $k_{\perp \bar{q}} \sim \Lambda_{\mathrm{QCD}}$ 《4, 5]. Thus the antiquark momentum $k_{\bar{q}}^{+}=z_{\bar{q}} q^{+} \simeq \Lambda_{\mathrm{QCD}}^{2} /\left(m_{N} x_{B}\right)$ stays finite in the Bjorken limit. The probability $\propto 1 / Q^{2}$ of the asymmetric photon splitting determines the scaling of the DIS cross section, $\sigma_{t o t}\left(\gamma^{*} p\right) \propto 1 / Q^{2}$. The non-perturbative scattering cross section $\sigma[(q \bar{q}) N] \sim 1 / \Lambda_{\mathrm{QCD}}^{2}$ of the $q \bar{q}$ Fock state corresponds to the quark distribution $f_{q / N}\left(x_{B}\right)$ in the $q^{-} \simeq 2 \nu$ (or Breit) frame.

The endpoint dynamics was also studied for quarkonium hadroproduction, $\pi N \rightarrow$ $J / \psi+X$ [6]. QCD factorization breaks down when the quarkonium carries large fractional momentum, $x_{F} \simeq 1-\Lambda_{\mathrm{QCD}}^{2} / M_{J / \psi}^{2}$. In this regime there is no hard scattering on a target parton (e.g., in a subprocess such as $g g \rightarrow c \bar{c}$ ). Rather, a compact Fock state in the pion projectile fluctuates into an endpoint state where nearly all momentum is carried by the heavy quark pair. The light valence quarks have transverse momenta of $\mathcal{O}\left(\Lambda_{\mathrm{QCD}}\right)$ and their soft, non-perturbative scattering in the target liberates the heavy quarks, which then appear in the final state.

According to Eq. (1.1) all endpoint configurations have short lifetimes in spite of their large transverse size. Like compact states they thus have a low number of constituents - in particular, comoving fields that have long formation times are absent. Hence there is no enhanced forward radiation in scattering processes.

The large size of endpoint configurations favors multiple scattering in the target. This enhances their importance in diffractive processes which require color singlet exchange. The aligned jet configuration contributes at leading twist to diffractive DIS, whereas multiple scattering of the compact, symmetric $q \bar{q}$ configurations is power suppressed. Endpoint configurations are also enhanced in scattering on nuclear targets due to the increased importance of multiple scattering. The nuclear dependence in effect measures the size of the contributing Fock states. The apparent absence of color transparency in large angle $e p \rightarrow e p$ [8] and $p p \rightarrow p p$ [9] scattering, with the target proton embedded in a nucleus, may signal dominant endpoint contributions in these processes ${ }^{1}$.

Quark helicity flip in hard photon and gluon interactions is suppressed by a factor $m_{q} / k_{\perp}$. Helicity is therefore conserved at leading twist in BL factorization. On the other hand, the low $k_{\perp} \sim \Lambda_{\mathrm{QCD}}$ of endpoint constituents implies that quark helicity flip is not suppressed. The relative importance of amplitudes with quark helicity flip is thus another measure of endpoint contributions.

The LC energy difference in (11.1) diverges when any fractional momentum $z_{i} \rightarrow$ 0 . This is the reason why distribution amplitudes, which are defined at equal LC time, vanish at the endpoints [1, 2]. As we emphasized above, however, target scattering is soft in the endpoint regime, implying a breakdown of LC dominance and of factorization into hard subprocess and distribution amplitudes. For this dynamics it is more natural to use the difference of ordinary energies in (1.1) which stays

\footnotetext{
${ }^{1}$ See Ref. [10] for a discussion and alternative explanations.
} 


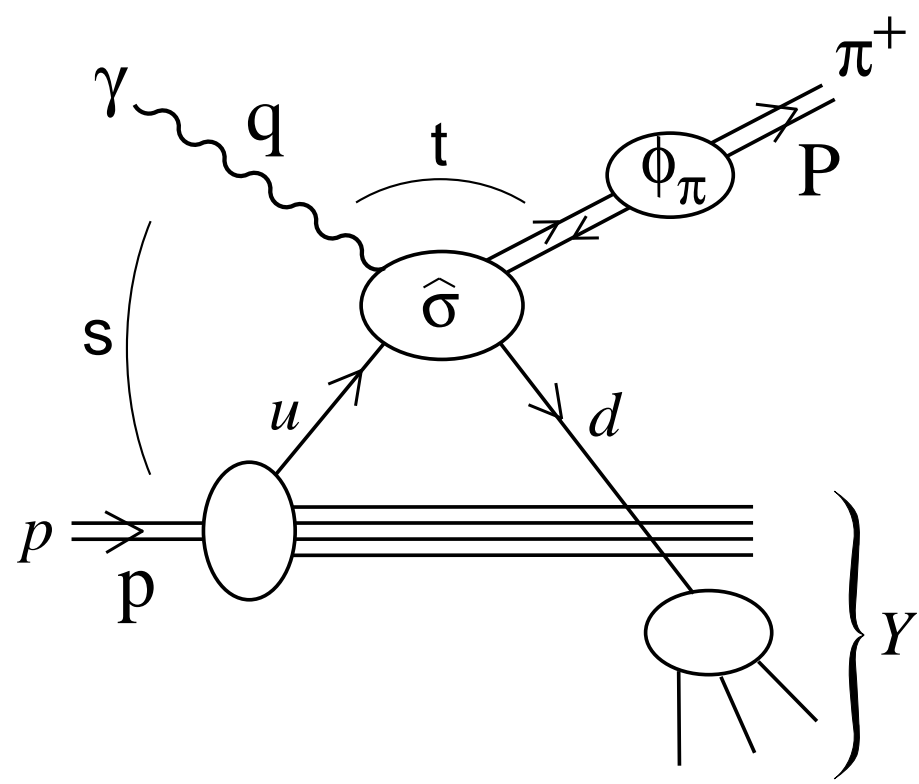

Figure 1: Semi-exclusive scattering. In the limit (2.1) the cross section factorizes into a hard subprocess cross section $\hat{\sigma}$ times a target parton distribution.

finite (albeit large) in the $z_{i} \rightarrow 0$ limit. This increases the importance of endpoint contributions in convolution integrals.

In this paper we study two processes where data indicates that endpoint contributions dominate. The perturbative QCD (PQCD) estimate for $d \sigma / d t\left(\gamma p \rightarrow \pi^{+} n\right)$, obtained from the semi-exclusive process $\gamma p \rightarrow \pi^{+} Y$ (Fig. 1) [11] using BloomGilman duality [12, 13, is two orders of magnitude below the data 114. In section 2 we show that the transverse size of the $\gamma u \rightarrow \pi^{+} d$ subprocess is effectively large, and that the color transparency assumed in the semi-exclusive process is thus likely to be violated.

Recent high energy data on $\rho^{0}$ and $\phi$ meson photoproduction show a dominance of quark helicity flip out to large momentum transfer $|t| \lesssim 12 \mathrm{GeV}^{2}$ [15]. In section 3 we study the properties of the $\gamma g \rightarrow \rho g$ subprocess amplitude. The amplitude for longitudinally polarized $\rho$ mesons (which conserves quark helicity) vanishes for real external photons. The amplitude for transversely polarized $\rho$ 's (which dominates in the data) has strongly enhanced endpoint contributions. Due to the suppression of quark helicity flip in hard scattering only the soft endpoint contributions can potentially explain the observed dimensional scaling of the cross section. We discuss qualitatively how the endpoint region might give rise to dimensional scaling.

\section{The size of $\gamma u \rightarrow \pi^{+} d$}

Our study of the $\gamma u \rightarrow \pi^{+} d$ process is motivated by its role as a subprocess of semiexclusive $\gamma p \rightarrow \pi^{+} Y$ scattering. Although no rigorous proof exists, the applicability 
of factorization is often assumed. Then, in the kinematic limit

$$
s \gg-t, M_{Y}^{2} \gg \Lambda_{\mathrm{QCD}}^{2}
$$

where ( $c f$. Fig. 1) $s=(q+p)^{2}=E_{\mathrm{CM}}^{2}$ and the invariant momentum transfer $t=$ $(q-P)^{2}$, the semi-exclusive cross section reads 11]

$$
\frac{d \sigma}{d t}\left(\gamma p \rightarrow \pi^{+} Y\right)=\sum_{q=u, \bar{d}} f_{q / p}(x) \frac{d \hat{\sigma}}{d t}\left(\gamma q \rightarrow \pi^{+} q^{\prime}\right) .
$$

The fractional momentum of the struck quark is $x=-t /\left(M_{Y}^{2}-t\right)$ and the subprocess cross section is given by $(\hat{s}=x s)$

$$
\frac{d \hat{\sigma}}{d t}\left(\gamma u \rightarrow \pi^{+} d\right)=\frac{256 \pi^{2} \alpha \alpha_{s}^{2}}{27 \hat{s}^{2}|t|}\left(e_{u}-e_{d}\right)^{2}\left[\int_{0}^{1} d z \frac{\Phi_{\pi}(z)}{z}\right]^{2} .
$$

Here $\Phi_{\pi}(z)$ is the pion distribution amplitude, i.e., its Fock state wave function for a $u \bar{d}$ pair at short transverse distance $\sim \mathcal{O}(1 / \sqrt{-t})$, with the $u$-quark carrying a fraction $z$ of the pion momentum. A color singlet $u \bar{d}$ pair of small transverse size does not rescatter in the proton target, giving the simple expression (2.2) for the semi-exclusive cross section.

There is as yet no data on $\gamma p \rightarrow \pi^{+} Y$ in the kinematic region (2.1). Assuming that semi-exclusive processes obey Bloom-Gilman duality, one may relate the $\gamma p \rightarrow$ $\pi^{+} Y$ cross section to the one for $\gamma p \rightarrow \pi^{+} n$. However, the measured $\gamma p \rightarrow \pi^{+} n$ cross section is so large that Bloom-Gilman duality would have to fail by two orders of magnitude for the prediction of the semi-exclusive cross section to be correct [14].

In light of the recent experimental evidence for Bloom-Gilman duality in inclusive reactions [13], such a gross failure seems rather unlikely. A more plausible explanation is that (2.2) is an underestimate of the true $\gamma p \rightarrow \pi^{+} Y$ cross section due to a lack of color transparency.

We shall use the photon virtuality as a probe of the transverse size of the subprocess (2.3). The cross section is independent of $Q^{2}$ when the size of the scattering region is small compared to $1 / Q^{2}$. We employ the asymptotic distribution amplitude

$$
\Phi_{\pi}(z)=\frac{\sqrt{6}}{2} f_{\pi} z(1-z)
$$

$\left(f_{\pi} \simeq 130 \mathrm{MeV}\right)$ and neglect quark masses $\left(m_{q}=0\right)$. In Fig. 2 we show the differential cross section $d \sigma / d t\left(\gamma_{\mathrm{T}}^{*}\left(Q^{2}\right) u \rightarrow \pi^{+} d\right)$ (solid line) for a transversely polarized virtual photon as a function the dimensionless ratio $Q^{2} /|t|$. While the real photon cross section given by (2.3) is finite, its slope at $Q^{2}=0$ is (as we shall see, logarithmically) infinite. Thus, however big the momentum transfer $|t|$ is, the transverse size of the photon scattering region remains large. For comparison we also show (dashed line in 


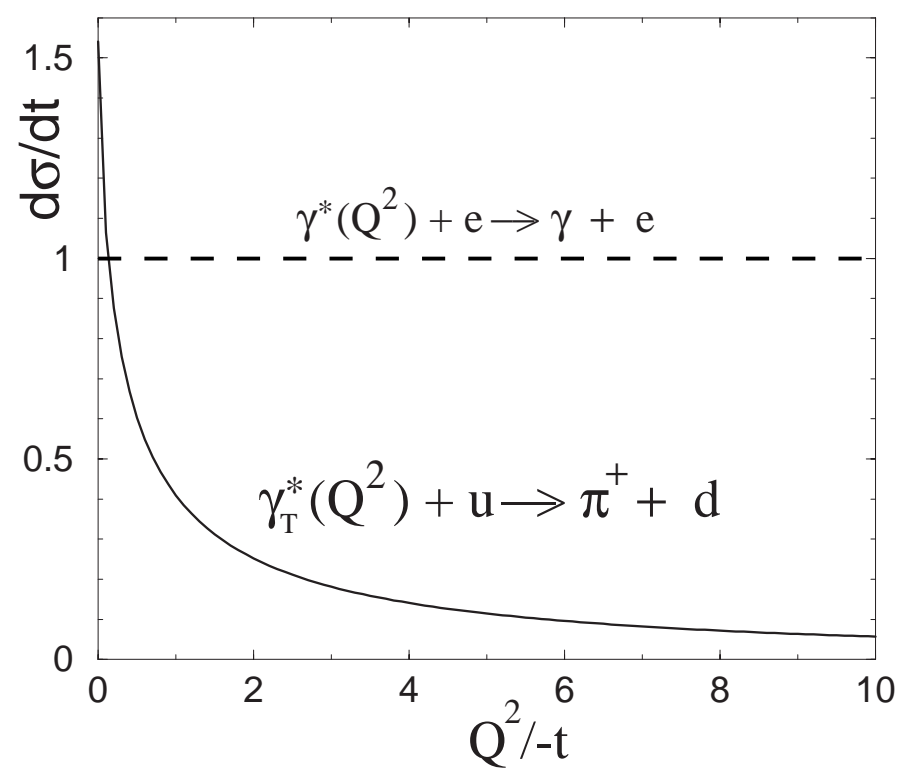

Figure 2: $d \sigma / d t\left(\gamma_{\mathrm{T}}^{*}\left(Q^{2}\right)+u \rightarrow \pi^{+}+d\right)$ for a transversely polarized photon as a function of $Q^{2} /|t|$ (solid line). For comparison, we show the corresponding plot for Compton scattering, $\gamma^{*}\left(Q^{2}\right)+e \rightarrow \gamma+e$ (dashed line). The normalization is arbitrary.

Fig. 2) that the Compton scattering $\gamma^{*} e \rightarrow \gamma e$ cross section is independent of $Q^{2}$ in the limit $s \gg|t|$, as expected due to the pointlike nature of the photon.

The divergent slope of the meson photoproduction cross section has already been noted by the authors of [16]. They concluded that the onset of the perturbative regime for the production of light vector mesons is at rather large momentum transfers, well beyond $30 \mathrm{GeV}^{2}$.

The strong $Q^{2}$ dependence of the $\gamma^{*} u \rightarrow \pi^{+} d$ cross section is due to endpoint contributions, even though the $z$-integral in $(2.3)$ is not enhanced near $z=0,1$. This may be seen from the expression for the scattering amplitude, which is a convolution of the pion distribution amplitude (2.4) with the $\gamma^{*} u \rightarrow(u \bar{d})+d$ subamplitude $\mathcal{H}_{\mu, \lambda \lambda^{\prime}}^{\nu \nu^{\prime}}$ (cf. Fig. 3a),

$$
\mathcal{M}_{\mu, \lambda \lambda^{\prime}}^{A B}\left(\gamma^{*}+u \rightarrow \pi^{+}+d\right)=\delta_{A B} \int_{0}^{1} d z \frac{1}{\sqrt{2}}\left[\mathcal{H}_{\mu, \lambda \lambda^{\prime}}^{+-}(z)-\mathcal{H}_{\mu, \lambda \lambda^{\prime}}^{-+}(z)\right] \Phi_{\pi}(z) .
$$

Here $A, B$ are the color indices of the incoming $u$ - and outgoing $d$-quark, $\mu$ is the photon helicity, $\lambda\left(\lambda^{\prime}\right)$ is the incoming $u$-quark (outgoing $d$-quark) helicity and $\nu\left(\nu^{\prime}\right)$ is the $u(\bar{d})$ quark helicity in the pair forming the $\pi^{+}$. For $m_{u}=m_{d}=0$ quark helicity is conserved ${ }^{2}$ and we shall only consider the $\mu=+1, \lambda=\lambda^{\prime}=-\frac{1}{2}$ amplitude. In the semi-exclusive limit (2.1) $\left(s \gg-t, Q^{2}\right)$,

$$
\mathcal{H}_{+,--}^{-+}=-\frac{2 \sqrt{2} e\left(4 \pi \alpha_{s}\right) C_{F}}{\sqrt{-t}}\left[\frac{e_{u}}{z-\bar{z} Q^{2} / t}-\frac{e_{d} \bar{z}}{z\left(\bar{z}-z Q^{2} / t\right)}\right],
$$

\footnotetext{
${ }^{2}$ Expressions for the helicity amplitudes for $Q^{2}, m_{q} \neq 0$ are given in the Appendix.
} 
where $C_{F}=\left(N_{c}^{2}-1\right) / 2 N_{c}$ is the color factor and $\bar{z}=1-z$. We make the following observations:

(a) At $Q^{2}=0$ the amplitude is $\propto\left(e_{u}-e_{d}\right) / z$. This endpoint behavior therefore arises both from the photon coupling to the 'slow' $u$-quark and to the 'fast' $d$-quark. On the other hand, the amplitude is finite for $z \rightarrow 1$ since the $u$-quark helicity $\nu=-\frac{1}{2}$ is opposite to that of the photon helicity $\mu=+1$. Thus the helicity flip between the projectile and fast outgoing particles is minimized.

(b) $d \mathcal{H} / d Q^{2} \propto e_{u} / z^{2}$ for $z \rightarrow 0$ at $Q^{2}=0$. The $1 / z^{2}$ behavior gives a logarithmic singularity in the convolution (2.5) when $\Phi_{\pi}(z) \propto z$. This is the origin of the infinite slope in Fig. 2. We also note that the singular contribution arises from the photon coupling to the slow quark.

This is distinct from the well known Feynman endpoint mechanism, where the photon couples to the fast quark.

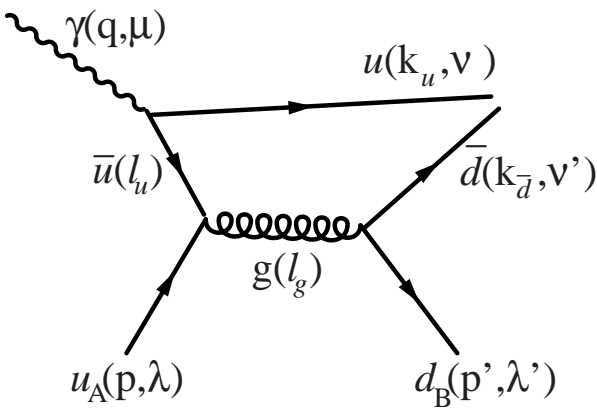

(a)

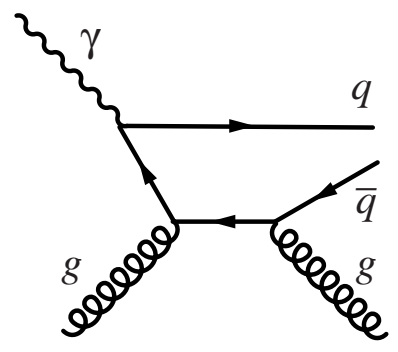

(b)

Figure 3: (a) A diagram contributing to $\gamma+u \rightarrow u \bar{d}+d$. (b) A diagram contributing to $\gamma+g \rightarrow q \bar{q}+g$.

The $Q^{2}$-sensitivity is due to the large transverse size of the $\gamma u \rightarrow \pi^{+} d$ process, as can be seen explicitly from the kinematics ( $c f$. Fig. 3a). We use the notation $v=\left[v^{+}, v^{-}, \mathbf{v}_{\perp}\right]$ where $v^{ \pm}=v^{0} \pm v^{3}$ for light-cone coordinates and take the photon momentum in the negative $z$-direction so that ${ }^{3}$

$$
\begin{aligned}
p & =\sqrt{s}\left[1,0, \mathbf{0}_{\perp}\right], \\
q & =\sqrt{s}\left[0,1, \mathbf{0}_{\perp}\right] \\
k_{u} & =\left[\frac{\left(z \mathbf{P}_{\perp}+\mathbf{k}_{\perp}\right)^{2}}{z \sqrt{s}}, z \sqrt{s}, z \mathbf{P}_{\perp}+\mathbf{k}_{\perp}\right] \\
k_{\bar{d}} & =\left[\frac{\left(\bar{z} \mathbf{P}_{\perp}-\mathbf{k}_{\perp}\right)^{2}}{\bar{z} \sqrt{s}}, \bar{z} \sqrt{s}, \bar{z} \mathbf{P}_{\perp}-\mathbf{k}_{\perp}\right]
\end{aligned}
$$

\footnotetext{
${ }^{3}$ For notational convenience we denote the (target) $u$-quark momentum by $p$ and the subprocess energy by $s$ rather than $\hat{s}$.
} 
for on-shell massless quarks. $\mathbf{P}_{\perp}$ denotes the pion's transverse momentum relative to the collision axis and $\mathbf{k}_{\perp}$ is the relative transverse momentum of its $u \bar{d}$ constituents. The pion momentum $P=k_{u}+k_{\bar{d}}$ satisfies $^{4} P^{2}=k_{\perp}^{2} / z \bar{z}$, where $k_{\perp}=\mathcal{O}\left(\Lambda_{\mathrm{QCD}}\right)$, and the momentum transfer $t=(q-P)^{2}=-P_{\perp}^{2}$.

The virtualities of the internal quark and gluon lines in Fig. 3a are

$$
\begin{aligned}
& \ell_{u}^{2}=\left(q-k_{u}\right)^{2}=-z P_{\perp}^{2}-2 \mathbf{P}_{\perp} \cdot \mathbf{k}_{\perp}-k_{\perp}^{2} / z \\
& \ell_{g}^{2}=\left(p+\ell_{u}\right)^{2}=\bar{z} s+\ell_{u}^{2}
\end{aligned}
$$

In the semi-exclusive limit (2.1) we see that $\ell_{u}^{2}$ becomes sensitive to $\mathbf{k}_{\perp}$ for

$$
z \lesssim \frac{k_{\perp}}{P_{\perp}} \sim \frac{\Lambda_{\mathrm{QCD}}}{\sqrt{-t}}
$$

Hence the subprocess is not transversally compact: the distance between the photon absorption and gluon emission vertices in Fig. 3a is given by the inverse of $\ell_{u \perp}=$ $\left|z \mathbf{P}_{\perp}+\mathbf{k}_{\perp}\right|=\mathcal{O}\left(\Lambda_{\mathrm{QCD}}\right)$ in the region (2.10). Moreover, BL factorization fails in this endpoint region since the hard subamplitude depends on the relative momentum of the quarks in the pion. These consequences of the kinematics imply the $Q^{2}$-sensitivity of the process.

We can also see why the large transverse size, i.e., the $Q^{2}$-sensitivity, arises only from the photon coupling to the slow quark. When the $u$-quark is fast, i.e., for $z \simeq$ $1-\Lambda_{\mathrm{QCD}} / \sqrt{-t}$, the transverse distance $1 / \ell_{u \perp}=\mathcal{O}(1 / \sqrt{|t|})$ while $\ell_{g}^{2} \propto s \Lambda_{\mathrm{QCD}} / \sqrt{-t}$ remains large since $s \gg-t$. Hence the distances between all interaction vertices in Fig. 3a are short for $z \rightarrow 1$.

The fact that the $Q^{2}$ derivative of the subprocess amplitude (2.6) is more endpoint sensitive than the the amplitude itself shows that a size measurement introduces inverse factors of $z$ and $1-z$. Our result does not change the fact that the $z$-integral of the leading twist cross section is flat (for the asymptotic distribution amplitude (2.4)), and thus is dominated by compact configurations. However, rescattering in the target will introduce dipole factors proportional to the transverse size and cause the convolution integral to be endpoint dominated. A failure of color transparency is thus a likely reason for the large discrepancy with data found in Ref. [14] for the $\gamma p \rightarrow \pi^{+} n$ cross section.

An analogous sensitivity to small photon virtualities can be observed for the photon-pion transition form factor [17, $\gamma^{*}\left(Q^{2}\right) \gamma^{(*)}\left(Q^{\prime 2}\right) \rightarrow \pi^{0}$ : its rate of change is logarithmically divergent as the ratio $Q^{\prime 2} / Q^{2} \rightarrow 0$. At $Q^{\prime 2}=0$ the pion transition form factor is given by the same integral over the distribution amplitude as appears in the cross section (2.3). The fact that the $\pi^{0}$ is produced in isolation and color

\footnotetext{
${ }^{4}$ If the $u$ and $\bar{d}$ quarks are not on-shell (as would be the case in the pion) we have $P^{2}=$ $\left(k_{\perp}^{2}+z k_{u}^{2}+\bar{z} k_{\bar{d}}^{2}\right) / z \bar{z}$. Hence an off-shellness of $\mathcal{O}\left(k_{\perp}^{2}\right)$ is sufficient to keep the pion on-shell even for $z \rightarrow 0,1$.
} 
transparency thus is not an issue may explain the phenomenological success (see [18 and references therein) of the PQCD prediction in this case.

We have checked that the Sudakov effect does not change our conclusions, by applying the modified factorization approach of Ref. [7] to the subprocess $\gamma u \rightarrow$ $\pi^{+} d$ in the above kinematical limit. In our numerical calculation we employed the asymptotic distribution amplitude (2.4), and two parametrizations of the transverse momentum dependence of the pion's LC wave function: a Gaussian of the form 19 $\exp \left[-a_{\pi}^{2} k_{\perp}^{2} /(z \bar{z})\right]$, with $a_{\pi} \simeq 0.86 \mathrm{GeV}^{-1}$ being the transverse size parameter, and a Gaussian of the form [20] $\exp \left[-\beta^{2}\left(k_{\perp}^{2}+m_{\text {eff }}^{2}\right) /(z \bar{z})\right]$ with an effective mass $m_{\text {eff }}=$ $0.33 \mathrm{GeV}$ and $\beta \simeq 0.94 \mathrm{GeV}^{-1}$. In both cases we found moderate Sudakov corrections of about $5-10 \%$, which shows that Sudakov effects do not play a significant role in the present discussion. Similar qualitative and quantitative conclusions about the impact of Sudakov corrections have been reached in Refs. [21, 17.

\section{Dimensional scaling with quark helicity flip in $\gamma+p \rightarrow \rho+Y$}

\subsection{The experimental evidence}

The ZEUS collaboration recently published [15] data on $\rho$ photoproduction, $\gamma+p \rightarrow$ $\rho+Y$, in the semi-exclusive kinematics specified by Eq. (2.1). The data cover $80<$ $\sqrt{s}<120 \mathrm{GeV}, 1.1<|t|<12 \mathrm{GeV}^{2}$ and is integrated over $x=-t /\left(M_{Y}^{2}-t\right) \gtrsim 0.01$. The scattering is believed to be dominantly diffractive and, due to the high value of $|t|$, to provide a testing ground for the BFKL exchange mechanism 22]. The hard subprocess is then $\gamma+g \rightarrow \rho+g$, with the t-channel containing a two-gluon ladder and the $\rho$ emerging via its distribution amplitude according to BL factorization.

However, the data [15] pose a serious challenge to this picture. Dimensional scaling predicts

$$
\frac{d \sigma(\gamma g \rightarrow \rho g)}{d t} \propto \frac{I_{\rho}^{2}}{|t|^{n}}
$$

with $n=3$. Here $I_{\rho}$ is an integral over the $\rho$ distribution amplitude with dimension $\mathrm{GeV}$. In contrast to the quark exchange cross section (2.3) there is no factor $s$ in the denominator of this gluon exchange cross section. The data agree with dimensional scaling, giving $n=3.21 \pm 0.04 \pm 0.15$ (in $\phi$ production the corresponding power is measured to be $n=2.7 \pm 0.1 \pm 0.2)$. Together with the fact that the $\phi / \rho$ cross section

ratio is consistent with the ratio $2 / 9$ of the charge factors for $|t| \gtrsim 4 \mathrm{GeV}^{2}$, this suggests that the $\gamma+g \rightarrow \rho+g$ process is hard and perturbative.

The upper part of the subprocess is shown in Fig. 3b. The quark pair produced at the photon vertex scatters off the two gluons and forms the vector meson via its distribution amplitude. The vector meson is expected to be longitudinally polarized since its quark and antiquark constituents have opposite helicities due to helicity conservation at the photon and gluon vertices. However, the ZEUS data show that 
the $\rho$ meson inherits (to a good approximation and in the full $t$-range) the transverse polarization of the incoming photon. In the BL factorization framework this implies a quark helicity flip, incurring an $m_{q}^{2} /|t|$ suppression factor in the cross section (3.1), which is then expected to scale with a power $n=4$.

Thus, we are faced with a dilemma. The data obeys simple dimensional scaling $(n=3)$, is consistent with the quark production process being hard $(\phi / \rho$ flavor symmetry indicates insensitivity to $m_{q}$ ) and the semi-exclusively produced $\rho$ meson carries both the momentum and the helicity of the projectile. But these attractive features are mutually inconsistent within the standard factorization framework [1] of exclusive processes.

The authors of 23] consider the possibility that the production of transverse vector mesons is due to a non-perturbative, chiral-odd wave function of the photon, which is proportional to the quark condensate. This contribution is subleading at asymptotically large momentum transfers. In such an approach the dimensional scaling observed [15] for $|t| \lesssim 12 \mathrm{GeV}^{2}$ would be accidental.

\subsection{The subprocess amplitudes}

To resolve the dilemma let us consider the structure of the factorized $\gamma+g \rightarrow \rho+g$ amplitude. It is a convolution of the quark pair production amplitude $\mathcal{G}(\gamma+g \rightarrow$ $q \bar{q}+g)$ and the $\rho$ distribution amplitude $\Phi_{\rho}^{\mu^{\prime}}$. For example, for transversely polarized $\left(\mu^{\prime}=+1\right) \rho$ mesons,

$$
\mathcal{M}_{\mu, \lambda \lambda^{\prime}}^{a b}(\gamma+g \rightarrow \rho+g)=\delta_{a b} \int_{0}^{1} d z \mathcal{G}_{\mu, \lambda \lambda^{\prime}}^{++}(z) \Phi_{\rho}^{+}(z)
$$

Here $a, b$ are gluon color indices, the $\mu, \lambda, \lambda^{\prime}$ indices of $\mathcal{G}$ are the helicities of the photon, incoming and outgoing gluon, respectively, and the upper indices refer to the $q$ and $\bar{q}$ helicities.

For simplicity we consider only the lowest order contribution to $\mathcal{G}(\gamma+g \rightarrow q \bar{q}+g)$ (cf. Fig. 3b). Higher order diagrams build the gluon ladder and are important for describing the $s$-dependence, but should not affect the helicity structure of the upper vertex, which is our present concern.

The transverse photon $(\mu=+1)$ amplitude with $\lambda=\lambda^{\prime}=+1$ and no quark helicity flip is

$$
\begin{aligned}
\mathcal{G}_{+,++}^{+-}(\gamma+g \rightarrow q \bar{q}+g) & =-\frac{\sqrt{2} e e_{q}\left(4 \pi \alpha_{s}\right)}{\sqrt{N_{c}} \sqrt{-t}} \frac{Q^{2}}{t} \\
& \times \frac{2 z-1}{\bar{z}\left(z-\bar{z} Q^{2} / t-m_{q}^{2} / t\right)\left(\bar{z}-z Q^{2} / t-m_{q}^{2} / t\right)} .
\end{aligned}
$$


This leading twist amplitude vanishes in photoproduction $\left(Q^{2}=0\right)$. The quark helicity flip amplitude contributing to transverse $\rho$ production in (3.2) is at ${ }^{5} Q^{2}=0$

$$
\begin{aligned}
\mathcal{G}_{+,++}^{++}(\gamma+g \rightarrow q \bar{q}+g) & =-\frac{\sqrt{2} e e_{q}\left(4 \pi \alpha_{s}\right)}{\sqrt{N_{c}} \sqrt{-t}} \frac{\sqrt{m_{q}^{2} /(-t)}}{z \bar{z}\left(z-m_{q}^{2} / t\right)\left(\bar{z}-m_{q}^{2} / t\right)} \\
& =-\frac{\sqrt{2} e e_{q}\left(4 \pi \alpha_{s}\right)}{\sqrt{N_{c}} \sqrt{-t}} \frac{\sqrt{m_{q}^{2} /(-t)}}{(z \bar{z})^{2}}\left[1+\mathcal{O}\left(\frac{m_{q}^{2}}{t}\right)\right] .
\end{aligned}
$$

The factor $(z \bar{z})^{2}$ in the denominator enhances the endpoint regions $z=0,1$ in the convolution (3.2) causing a (logarithmic) singularity in the $z$-integral for distribution amplitudes which vanish linearly at the endpoints. This implies a breakdown of factorization in semi-exclusive $\rho$ photoproduction.

\subsection{Endpoint behavior of the distribution amplitude}

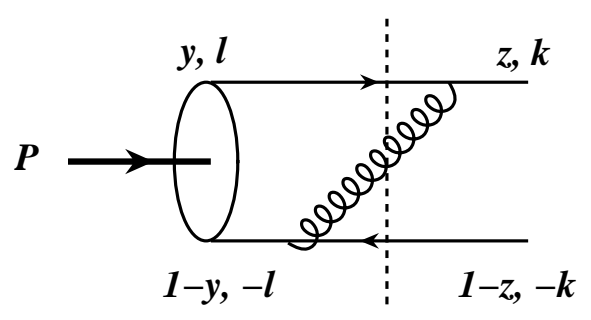

Figure 4: A representative of the onegluon exchange diagrams contributing to the evolution equation in timeordered LC perturbation theory.
Even though factorization fails due to the strong enhancement of the subprocess amplitude in the region where one of the produced quarks carries nearly all the momentum, we may still see qualitatively how the experimentally observed dimensional scaling of the cross section can arise. As we emphasized above, this must be due to the endpoint contributions, since for finite values of $z$ the transverse $\rho$ cross section is suppressed by $m_{q}^{2} /|t|$, giving $n=4$ in Eq. (3.1).

The distribution amplitude $\Phi_{\rho}^{\mu^{\prime}}(z)$ appearing in the convolution (3.2) is the valence Fock state amplitude at equal light-cone $(L C)$ time. Its endpoint behavior can be determined from the evolution equation, which, at leading order, corresponds to one-gluon exchange diagrams, $c f$. Fig. 4 [1]. Thus, the wave function can be written as

$$
\Phi_{\rho}\left(z, \mu_{F}\right)=\int d y \int^{\mu_{F}} \frac{d^{2} \mathbf{l}_{\perp}}{16 \pi^{3}} \alpha_{s}\left(\mu_{F}\right) \frac{\mathcal{S}\left(y, z, \mathbf{l}_{\perp}, \mathbf{k}_{\perp}\right)}{P^{-}-\sum_{i} p_{i}^{-}+i \epsilon} \Phi_{\rho}\left(y, \mathbf{l}_{\perp}\right),
$$

where $P^{-}$is the meson LC energy and the sum is over all LC energies $p_{i}^{-}$of the intermediate $q \bar{q} g$ state. $\mathcal{S}$ is a momentum dependent function which is finite at the endpoints. Hence, the behavior for $z \rightarrow 1$ follows from the energy denominator of the intermediate state,

$$
P^{-}-\sum_{i} p_{i}^{-} \simeq-\frac{k_{\perp}^{2}+m_{q}^{2}}{(1-z) P^{+}} \quad \text { for } \quad z \rightarrow 1 .
$$

\footnotetext{
${ }^{5}$ Expressions for $Q^{2} \neq 0$ are given in the Appendix.
} 
An analogous behavior can be found for $z \rightarrow 0$. Consequently, $\Phi_{\rho}\left(z, \mu_{F}\right)$ vanishes at $z=0,1$ because the LC energy of a parton with momentum fraction $z$ tends to infinity as $z \rightarrow 0$. However, as we saw in the previous section, the subprocess is soft and therefore not light-cone dominated for $z \lesssim z_{s} \simeq \Lambda_{\mathrm{QCD}} / \sqrt{-t}$. The LC energy is not relevant in this region - while the ordinary energy difference (11.1) obviously remains finite and is of $\mathcal{O}\left(\Lambda_{\mathrm{QCD}}\right)$ as the longitudinal momentum of a parton vanishes.

This suggests that $\Phi_{\rho}^{\mu^{\prime}}(z)$ (effectively) does not vanish at the endpoints. The $z$-integral is then linearly divergent at $z=0,1$. Truncating the integration region where the subprocess becomes soft we get

$$
\int_{z_{s}}^{1-z_{s}} \frac{\Phi_{\rho}^{\mu^{\prime}}(z)}{z^{2} \bar{z}^{2}} \propto \frac{\sqrt{-t}}{\Lambda_{\mathrm{QCD}}} \Phi_{\rho}^{\mu^{\prime}}(0) .
$$

Thus we gain a factor $\sqrt{-t}$ in the amplitude, which restores dimensional scaling, implying $n=3$ in Eq. (3.1). As already remarked above, here we only give qualitative arguments for the observed scaling. In order to predict the normalization of the cross section a more detailed analysis is necessary, which is however beyond the scope of the present work.

\section{Summary}

In this paper we have attempted to give a physical picture able to explain some qualitative aspects of the data on meson production at large momentum transfer $|t|$ by real, transversely polarized photons. Also, we have pointed out why some expectations based on the common assumption of factorization in exclusive PQCD studies seem to fail. Namely, we saw that the scattering is likely to be endpoint dominated and thus involve $q \bar{q}$ pairs of large transverse size. Hence it appears that highly asymmetric Fock states, where one quark carries nearly all the momentum, do have a significant overlap with hadron wave functions. This would explain the apparent absence of color transparency in $e A \rightarrow e p(A-1)$ [ ] and $p A \rightarrow p p(A-$ 1) [9].

Endpoint contributions are enhanced in photoproduction since the wave function of transverse photons does not vanish at $z=0,1$. This also prevents factorization of the transverse photon amplitude in deeply virtual meson production at high $Q^{2}$ and low $|t|$ [24]. The longitudinal photon wave function $\Psi_{\gamma}^{\mu=0}(z) \propto z(1-z)$ favors contributions from $q \bar{q}$ pairs of small transverse size $\sim 1 / Q$. The color transparency observed in $\gamma^{*}\left(Q^{2}\right)+N \rightarrow \rho+N$ [25, 26, 27] agrees with this.

Due to the short lifetime of the endpoint states their dynamics has many of the attributes of hard scattering, despite their large transverse size and soft scattering in the target. We have found that the Sudakov form factor numerically leads to a sup-

pression of no more than about $10 \%$, and is thus of minor importance. Furthermore, 
we have presented qualitative arguments suggesting that endpoint contributions may explain the dimensional scaling of the ZEUS data. The endpoint states are not color transparent, nor do they preserve quark helicity. These features make it possible to identify their contribution to exclusive processes.

\section{Acknowledgments}

It is a pleasure to thank Stan Brodsky and Jim Crittenden for many helpful discussions and comments on the manuscript. This work was begun while PH was employed by Nordita, and has been supported by the European Commission under contract HPRN-CT-2000-00130 and by the Academy of Finland under project 102046.

\section{A. Appendix}

\section{A.1 Quark production amplitudes}

We present the helicity amplitudes $\mathcal{H}_{\mu, \lambda \lambda^{\prime}}^{\nu \nu^{\prime}}$ for the subprocess $\gamma^{*}(\mu) u(\lambda) \rightarrow u(\nu) \bar{d}\left(\nu^{\prime}\right)+$ $d\left(\lambda^{\prime}\right)$ for $Q^{2}, m_{q} \neq 0$. For transversely polarized photons we find

$$
\begin{aligned}
\mathcal{H}_{+,++}^{+-}=-\mathcal{H}_{-,--}^{-+} & =\frac{2 \sqrt{2} e\left(4 \pi \alpha_{s}\right) C_{F}}{\sqrt{-t}} \\
& \times\left[\frac{e_{u} z}{\bar{z}\left(z-\bar{z} Q^{2} / t-m_{q}^{2} / t\right)}-\frac{e_{d}}{\left(\bar{z}-z Q^{2} / t-m_{q}^{2} / t\right)}\right] .
\end{aligned}
$$

The amplitudes $\mathcal{H}_{-,++}^{+-}$and $\mathcal{H}_{+,--}^{-+}$are obtained from $\mathcal{H}_{+,++}^{+-}$and $\mathcal{H}_{-,--}^{-+}$, respectively, by exchanging $z \rightarrow \bar{z}, e_{u} \leftrightarrow e_{d}$ and reversing the overall sign. For longitudinally polarized photons we get

$$
\mathcal{H}_{0,++}^{+-}=\mathcal{H}_{0,--}^{-+}=\frac{4 e\left(4 \pi \alpha_{s}\right) C_{F}}{\sqrt{-t}}\left[\frac{e_{u} \sqrt{Q^{2} /(-t)}}{z-\bar{z} Q^{2} / t-m_{q}^{2} / t}+\frac{e_{d} \sqrt{Q^{2} /(-t)}}{\bar{z}-z Q^{2} / t-m_{q}^{2} / t}\right] .
$$

The quark helicity flip amplitudes read

$$
\mathcal{H}_{+,--}^{++}=\mathcal{H}_{-,++}^{--}=-\frac{2 \sqrt{2} e\left(4 \pi \alpha_{s}\right) C_{F}}{\sqrt{-t}}\left[\frac{e_{u} \sqrt{m_{q}^{2} /(-t)}}{z \bar{z}\left(z-\bar{z} Q^{2} / t-m_{q}^{2} / t\right)}\right] .
$$

For $\mathcal{H}_{+,++}^{++}$and $\mathcal{H}_{-,--}^{--}$we have to make the replacements $z \rightarrow \bar{z}, e_{u} \rightarrow e_{d}$ in the above respective amplitudes. All other helicity amplitudes vanish in the kinematical limit (2.1). The helicity non-flip amplitudes agree with those given in Ref. 28] in the limit of large c.m. energies and when appropriate replacements for the charge factors are made. ${ }^{6}$ We note that in the helicity flip amplitudes (A.3) the photon couples

\footnotetext{
${ }^{6}$ In Ref. 28] the production of flavor-neutral mesons is considered. Also note that the normalization of the subprocess amplitudes is different than in our case.
} 
only to the quark whose helicity is flipped. According to (2.8) the gluon virtuality in Fig. 3a is of order $s$. Hence to leading order in the limit (2.1) the quark helicity can flip only at the photon vertex.

\section{A.2 Gluon production amplitudes}

The helicity amplitudes for the subprocess $\gamma^{*}(\mu)+g(\lambda) \rightarrow q(\nu) \bar{q}\left(\nu^{\prime}\right)+g\left(\lambda^{\prime}\right)$ are denoted by $\mathcal{G}_{\mu, \lambda \lambda^{\prime}}^{\nu \nu^{\prime}}$. For transversely polarized photons we find

$$
\begin{aligned}
\mathcal{G}_{+,++}^{+-}=-\mathcal{G}_{-,++}^{-+} & =-\frac{\sqrt{2} e e_{q}\left(4 \pi \alpha_{s}\right)}{\sqrt{N_{\mathrm{c}}} \sqrt{-t}} \frac{Q^{2}}{t} \\
& \times \frac{2 z-1}{\bar{z}\left(z-\bar{z} Q^{2} / t-m_{q}^{2} / t\right)\left(\bar{z}-z Q^{2} / t-m_{q}^{2} / t\right)},
\end{aligned}
$$

and for longitudinally polarized photons

$$
\mathcal{G}_{0,++}^{+-}=\mathcal{G}_{0,++}^{-+}=\frac{2 e e_{q}\left(4 \pi \alpha_{s}\right)}{\sqrt{N_{\mathrm{c}}} \sqrt{-t}} \frac{\sqrt{Q^{2} /(-t)}\left(1-Q^{2} / t\right)}{\left(z-\bar{z} Q^{2} / t-m_{q}^{2} / t\right)\left(\bar{z}-z Q^{2}-m_{q}^{2} / t\right)} .
$$

The amplitudes $\mathcal{G}_{+,++}^{-+}$and $\mathcal{G}_{-,++}^{+-}$are obtained from $\mathcal{G}_{+,++}^{+-}$and $\mathcal{G}_{-,++}^{-+}$respectively by replacing $z \rightarrow 1-z$. For the quark helicity flip amplitudes we obtain

$$
\begin{aligned}
\mathcal{G}_{+,++}^{++}=\mathcal{G}_{-,++}^{--} & =-\frac{\sqrt{2} e e_{q}\left(4 \pi \alpha_{s}\right)}{\sqrt{N_{\mathrm{c}}} \sqrt{-t}} \frac{\sqrt{m_{q}^{2} /(-t)}}{z \bar{z}} \\
& \times \frac{1-Q^{2} / t}{\left(z-\bar{z} Q^{2} / t-m_{q}^{2} / t\right)\left(\bar{z}-z Q^{2} / t-m_{q}^{2} / t\right)} .
\end{aligned}
$$

For the above combination of quark and photon helicities, the set of amplitudes with negative gluon helicities is identical, i.e.,

$$
\mathcal{G}_{\mu,--}^{\nu \nu^{\prime}}=\mathcal{G}_{\mu,++}^{\nu \nu^{\prime}} .
$$

All other helicity amplitudes vanish in the limit (2.1). Again, we find agreement with the results given in Ref. [28].

\section{References}

[1] G. P. Lepage and S. J. Brodsky, Phys. Rev. D 22 (1980) 2157.

[2] S. J. Brodsky and G. P. Lepage, In Perturbative Quantum Chromodynamics, (A. H. Mueller, Ed.) World Scientific (1989), p. 23-240 (SLAC-PUB-4947).

[3] N. Isgur and C. H. Llewellyn Smith, Phys. Rev. Lett. 52 (1984) 1080 and Nucl. Phys. B 317 (1989) 526;

A.V. Radyushkin Nucl. Phys. A 527 (1991) 1530 and Nucl. Phys. A 532 (1991) 141d. 
[4] J. D. Bjorken, J. B. Kogut and D. E. Soper, Phys. Rev. D 3 (1971) 1382.

[5] S. J. Brodsky, P. Hoyer and L. Magnea, Phys. Rev. D 55 (1997) 5585 hep-ph/9611278.

[6] S. J. Brodsky, P. Hoyer, A. H. Mueller and W.-K. Tang, Nucl. Phys. B 369 (1992) 519 .

[7] J. Botts and G. Sterman, Nucl. Phys. B 325 (1989) 62;

H.-N. Li and G. Sterman, Nucl. Phys. B 381 (1992) 129.

[8] T. G. O'Neill et al., Phys. Lett. B 351 (1995) 87 hep-ph/9408260.

[9] A. Leksanov et al., Phys. Rev. Lett. 87 (2001) 212301 hep-ex/0104039.

[10] M. M. Sargsian et al., J. Phys. G 29 (2003) R1 nucl-th/0210025.

[11] S. J. Brodsky, M. Diehl, P. Hoyer and S. Peigne, Phys. Lett. B 449 (1999) 306 hep-ph/9812277.

[12] E. D. Bloom and F. J. Gilman, Phys. Rev. Lett. 25 (1970) 1140 and Phys. Rev. D 4 (1971) 2901.

[13] I. Niculescu et al., Phys. Rev. Lett. 85 (2000) 1182 and 1186.

A. Airapetian et al. [HERMES Collaboration], Phys. Rev. Lett. 90 (2003) 092002 hep-ex/0209018.

[14] P. Eden, P. Hoyer and A. Khodjamirian, J. High Energy Phys. 0110 (2001) 040 hep-ph/0110297.

[15] S. Chekanov et al. [ZEUS Collaboration], Eur. Phys. J. C 26 (2003) 389 hep-ex/0205081.

[16] I. F. Ginzburg and D. Y. Ivanov, Phys. Rev. D 54 (1996) 5523, hep-ph/9604437].

[17] M. Diehl, P. Kroll and C. Vogt, Eur. Phys. J. C 22 (2001) 439 hep-ph/0108220.

[18] J. Gronberg et al. [CLEO Collaboration], Phys. Rev. D 57 (1998) 33 hep-ex/9707031.

[19] R. Jakob and P. Kroll, Phys. Lett. B 315, 463 (1993).

[20] S. J. Brodsky, T. Huang and G. P. Lepage, Particles and Fields 2, eds. Z. Capri and A.N. Kamal (Banff Summer Institute) p. 143 (1983).

[21] P. Jain, B. Pire and J. P. Ralston, Phys. Rept. 271, 67 (1996) [hep-ph/9511333].

[22] J. R. Forshaw and M. G. Ryskin, Z. Physik C 68 (1995) 137 hep-ph/9501376;

J. Bartels, J. R. Forshaw, H. Lotter and M. Wusthoff, Phys. Lett. B 375 (1996) 301 hep-ph/9601201. 
[23] D. Y. Ivanov, R. Kirschner, A. Schafer and L. Szymanowski, Phys. Lett. B 478 (2001) 101 [Erratum-ibid. B 498 (2001) 295] hep-ph/0001255.

[24] J. C. Collins, L. Frankfurt and M. Strikman, Phys. Rev. D 56 (1997) 2982 hep-ph/9611433.

[25] M. R. Adams et al. [E665 Collaboration], Phys. Rev. Lett. 74 (1995) 1525.

[26] M. Arneodo et al. [New Muon Collaboration], Nucl. Phys. B 429 (1994) 503.

[27] K. Ackerstaff et al. [HERMES Collaboration], Phys. Rev. Lett. 82 (1999) 3025 hep-ex/9811011;

A. Airapetian [HERMES Collaboration], Phys. Rev. Lett. 90 (2003) 052501 hep-ex/0209072.

[28] H. W. Huang and P. Kroll, Eur. Phys. J. C 17 (2000) 423 hep-ph/0005318. 